\title{
Ventricular tachycardia?
}

Beatrice Kühn $^{1 *}$, Stefan Borov ${ }^{1}$, Bernhard Zrenner ${ }^{1}$

${ }^{1}$ Chief physician at the Clinic for Cardiology, Pneumology and Internal Intensive Care Medicine LAKUMED Klinikum Landshut-Achdorf

*Corresponding Author: Beatrice Kühn, Chief physician at the Clinic for Cardiology, Pneumology and Internal Intensive Care Medicine LAKUMED Klinikum Landshut-Achdorf

Received Date: May 29, 2021; Accepted Date: July 05, 2021; Published Date: July 09, 2021

Citation: Beatrice Kühn, Stefan Borov, Bernhard Zrenner, (2021) Ventricular tachycardia?. J. Clinical Cardiology and Cardiovascular Interventions, 4(13); Doi:10.31579/2641-0419/188

Copyright: () 2021 Beatrice Kühn, This is an open-access article distributed under the terms of the Creative Commons Attribution License, which permits unrestricted use, distribution, and reproduction in any medium, provided the original author and source are credited.

A 59-year-old patient presented with "suspicion of ventricular tachycardia" because of "abnormalities in the long-term ECG" (Figure.
1). Cryoablation of the slow path had already taken place beforehand, in the case of slow-fast atrioventricular nodal reentry tachycardia.

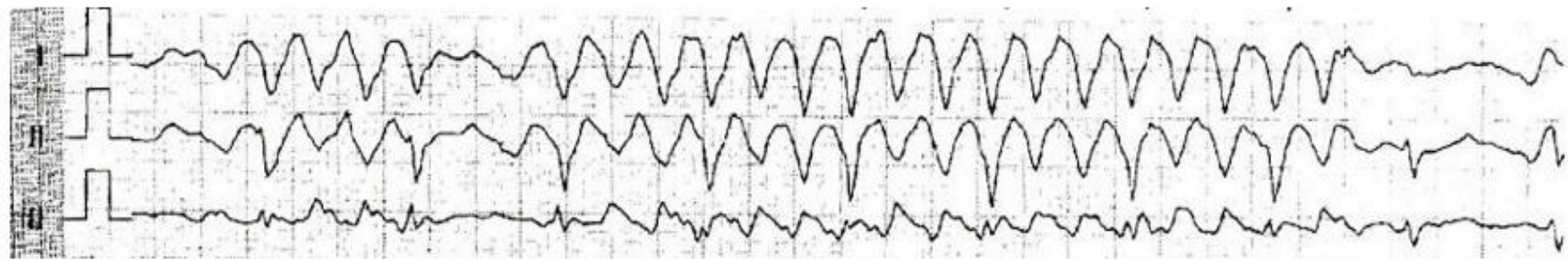

Figure 1: GP long-term ECG

The ECG on the ward showed no evidence of long or short QT (cQT time of $430 \mathrm{~ms}$ ), a heart rate of 86 / min with sinus rhythm.
The family doctor's long-term EKG also showed a hidden sinus rhythm (Figure. 2). Furthermore, these artifacts only show up in the limb leads, not also in the chest wall leads.

A more detailed anamnesis of the event showed that the patient was operating an electrical mixer at the time of the said ventricular tachycardia and was completely asymptomatic.

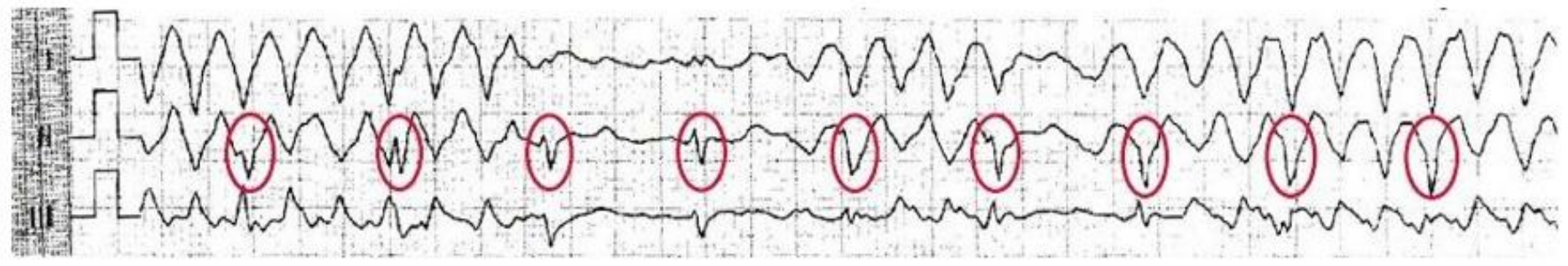

Figure 2: More detailed analysis of the family doctor's long-term ECG

We simulated the situation on the ward. Here the same artifacts appeared while the patient was operating the mixer. This can be generated by the electrical impulses transmitted to the body in the form of these artifacts.
The use of any small electronic device that emits strong vibrations can be a disruptive factor in the diagnosis of intermittent tachycardias. 


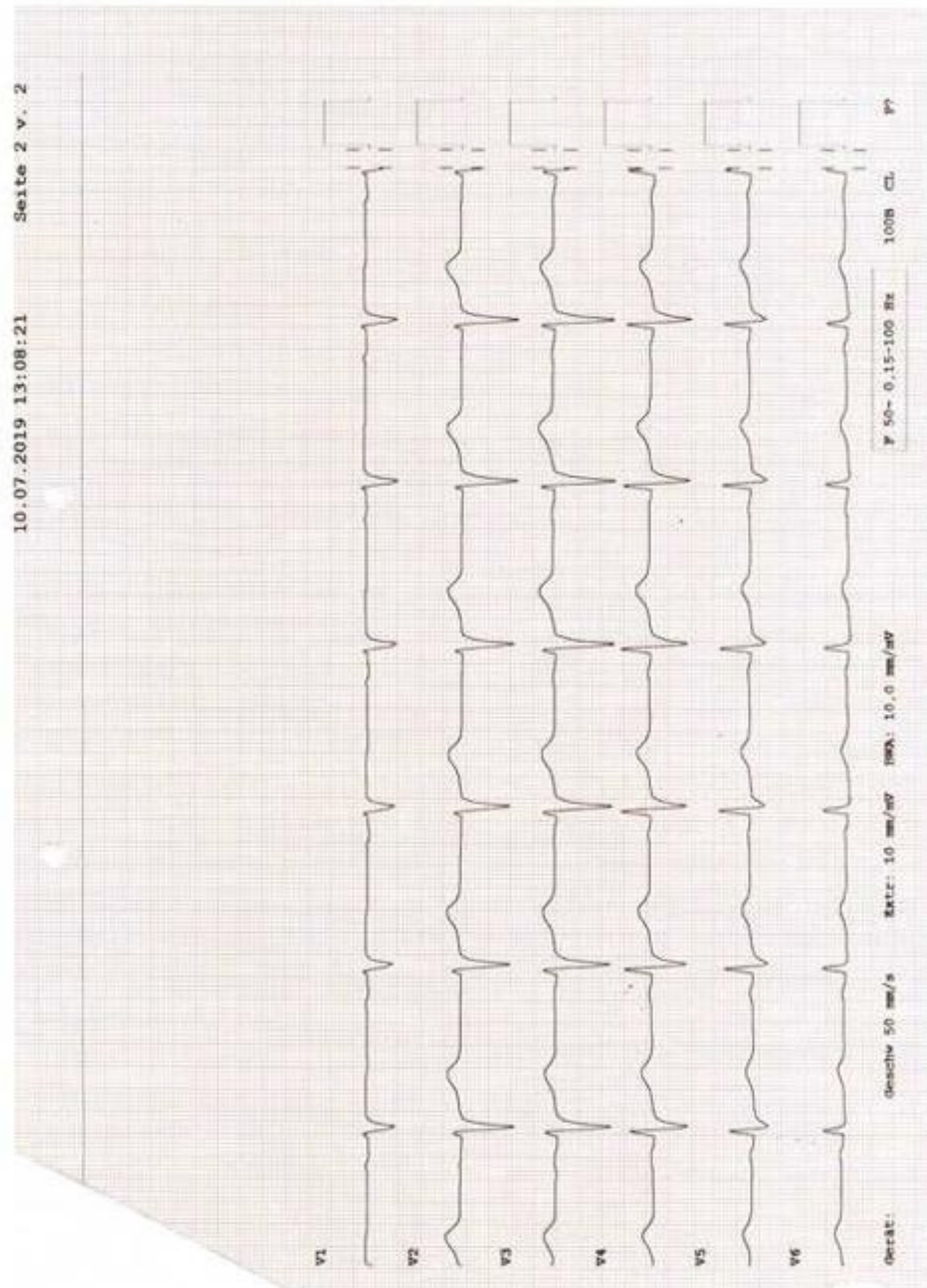




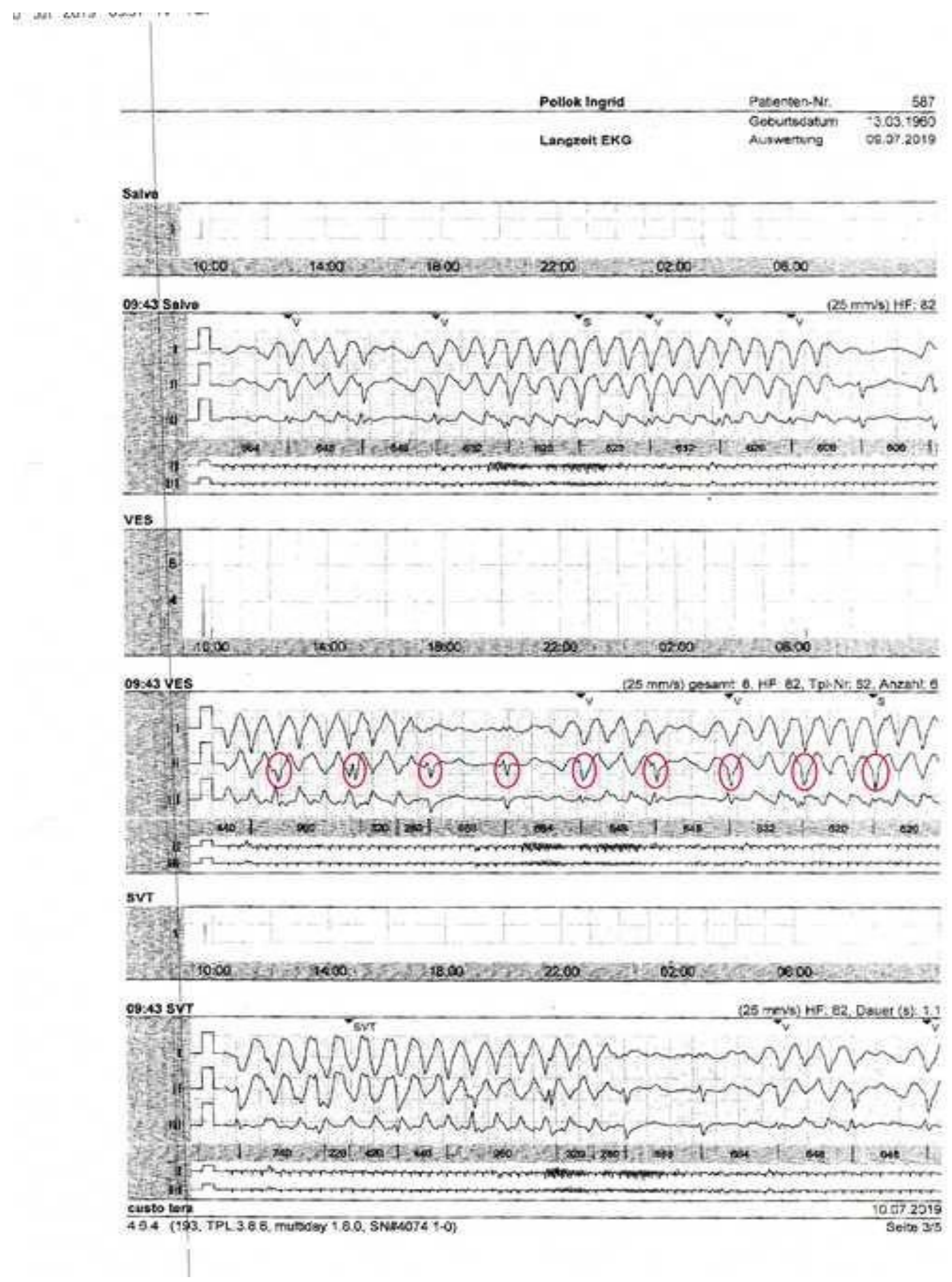

